\title{
Evaluation of the ability of the C-reactive protein-to-albumin ratio to predict short-term mortality in patients with COVID-19
}

\author{
Serdar Özdemir ${ }^{1}$, Abdullah Algin ${ }^{1,2}$ \\ ${ }^{1}$ University of Health Sciences Turkey, Ümraniye Training and Research Hospital, Clinic of Emergency Medicine, Istanbul, Turkey \\ ${ }^{2}$ University of Health Sciences Turkey, Somalia Mogadishu Recep Tayyip Erdogan Faculty of Health Sciences, Mogadishu, Somalia
}

Received: 2021-10-12.

Accepted: 2021-11-04

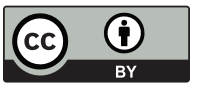

This work is licensed under a Creative Commons Attribution 4.0 International License

\section{J Clin Med Kaz 2021; 18(6):35-39}

Corresponding author:

Serdar Özdemir.

E-mail: dr.serdar55@hotmail.com;

ORCID: 0000-0002-6186-6110

\section{Abstract}

Objective: This study aimed to determine the ability of the C-reactive protein (CRP)-to-albumin ratio (CAR) to predict short-term mortality in patients with COVID-19.

Material and methods: This retrospective, observational, cohort study included patients with COVID-19. The patients' demographics, clinical characteristics, CRP, albumin, CAR, blood urea nitrogen, creatinine, highsensitive cardiac troponin I and all-cause mortality within 30 days after admission were noted. The receiver operating characteristic curve analysis was performed, and odds ratios (OR) were calculated to determine the discriminative ability of the parameters.

Results: A total of 103 patients with a median of age of 57 (25th - 75th percentiles: 32-76) years were included in the study. The rate of 30-day mortality was $4.8 \%$ for the study cohort. According to the best Youden's index, the cutoff value for CRP was determined as 66.67 (sensitivity: 80\%, specificity: 78.6\%), and the area under curve (AUC) value was 0.801 (95\% confidence interval $[\mathrm{Cl}$ ]: 71.1-87.3). According to the best Youden's index, the cut-off value for CAR was 0.18 (sensitivity: 80\%, specificity: 78.6\%), and the AUC value was 0.806 (95\% Cl: 71.6-87.7). There was no statistically significant difference between the AUC values of CRP and CAR (DeLong equality test, $p=0.938)$. The OR of CRP $(>66.67$ $\mathrm{mg} / \mathrm{L}$ ) and CAR (>0.18) for 30-day mortality were 14.667 (95\% Cl: 1.555-138.299) and 13.818 (95\% Cl: 1.468-130.076), respectively.

Conclusion: CAR was not useful in predicting 30-day mortality in patients with COVID-19. The calculation of CAR rather than CRP had no clinically significant contribution to the prediction of 30-day mortality in this patient group.

Key words: COVID-19, SARS-Cov-2, blood tests, coronavirus, C-reactive proteins, albumin, laboratory

\section{Introduction}

Severe acute respiratory syndrome coronavirus 2 (SARS-CoV-2), a respiratory RNA virus that causes severe acute respiratory failure emerged as an epidemic in Wuhan, China at the end of 2019 [1]. It turned into a pandemic, spreading across the world and negatively affecting social lives, economies, and health systems of countries [2]. From March 2020, when the disease was declared a pandemic to June 2021, it infected more than 180 million people and caused the deaths of more than 3.5 million people.

With the spread of the disease around the world, many researchers have investigated the course of the disease and prognostic factors to use resources effectively [3-5]. It has been shown that an increase in inflammatory markers, such as C-reactive protein (CRP), interleukin-6, leucocyte count, and erythrocyte sedimentation rate can be a marker of critical illness and mortality $[3,4,6]$. On the other hand, it has also been suggested that a decrease in markers, such as lymphocyte count and albumin can also be used in the detection of critical patients and predicting mortality. Researchers constantly attempt to identify better markers $[5,6]$. In order to achieve better predictability, hematological ratios, including neutrophil-to-lymphocyte, lymphocyte-to-CRP, lymphocyte-to-monocyte and platelet-to-lymphocyte ratios have been previously studies [5-7]. Acute-phase proteins (APPs) are proteins whose levels fluctuate in response to tissue injury, for example, trauma, acute infection, chronic inflammation, myocardial infarction, and malignancy. Their concentrations increase (positive APPs) or decrease (negative APPs) in response to the acute-phase reaction [8]. Based on the results reported in the literature, we considered that a ratio with a positive 
APP in the numerator and a negative APP in the denominator might be a near-ideal predictor [3-8]. Therefore, we chose CRP as a positive APP and albumin as a negative APP. In this study, we aimed to determine the ability of the CRP-to-albumin ratio (CAR) to predict short-term mortality in patients with coronavirus disease 2019 (COVID-19).

\section{Material and methods Study design}

This retrospective cohort study was conducted at Ümraniye Training and Research Hospital, a 695-bed tertiary education hospital with 1,110 daily patient admissions (annual average) to the emergency department (ED). The data of the patients admitted to our pandemic clinics between April 1, 2020 and May 1,2020 were collected retrospectively.

\section{Study population}

Our study population consisted of patients who were admitted to the pandemics clinic with the suspicion of COVID-19 between April 1, 2020 and May 1, 2020. All the patients with a positive test result for SARS-CoV-2, who were also tested for CRP and albumin, were included in the study. Patients chronic liver diseases were excluded from the study considering that their baseline albumin levels could be lower independent of COVID-19. The hospitalization and intensive care admission decisions of the patients were made by the emergency medicine specialist. The follow-up of the hospitalized patients was performed by a pulmonologist or an infectious disease specialist or an internist. The hospitalization decision and treatment planning were made in accordance with the COVID-19 Outbreak Management and Working Guideline of the Turkish Ministry of Health.

\section{Data collection}

The patients' demographics, clinical characteristics (included comorbidities and symptoms), vital parameters on admission laboratory findings, and emergency department outcomes were obtained from the hospital computer-based patient data system and analyzed by the researchers. Comorbidities were recorded as coronary artery disease, diabetes mellitus, chronic obstructive pulmonary diseases, hypertension, congestive heart failure, chronic renal failure, and malignancy. The symptoms of the disease were recorded as fever, cough, sputum, dyspnea, weakness, muscle-joint pain, loss of smell or taste, headache, sore throat, vomiting-nausea, and diarrhea. Systolic blood pressure, diastolic blood pressure, pulse pressure, body temperature, respiratory rate, and peripheral oxygen saturation were recorded as vital parameters. The documented laboratory parameters were blood urea nitrogen (BUN), creatinine, CRP, D-dimer, highsensitive cardiac troponin I (cTnI), CAR, albumin, white blood cell count, neutrophil count, lymphocyte count, platelet count, mean platelet volume, and mean corpuscular volume. The CAR values were calculated by two researchers.

To confirm SARS-CoV-2, the ORF1ab and $\mathrm{N}$ genes of SARS-CoV-2 were examined, and the Biorad CFX 96 platform was used. Ct values of 29 and above were considered as positive. The cases with a positive result for both genes were reported as SARS-CoV-2-positive.

\section{Statistical analysis}

IBM Corp. Released 2020. IBM SPSS Statistics for Mac, Version 27.0. Armonk, NY: IBM Corp. was used to perform statistical analyses. To assess the conformance of variables to normal distribution, the Kolmogorov-Smirnov test was conducted. The data that matched the normal distribution were presented with mean and standard deviation values, and the remaining data were expressed as interquartile range and median values. Categorical data were presented with percentages and number of cases. For the comparison of qualitative and quantitative data between two groups, the Mann-Whitney U and chi-square tests were used. We also constructed a receiveroperating characteristic curve (ROC) for short-term mortality and obtained the area under the curve (AUC) values for individual variables using the MedCalc Statistical Software version 12.7.7 (MedCalc Software by, Ostend, Belgium; http://www.medcalc. org; 2013). The AUC values of the laboratory findings were calculated and tested mutually for significance with the DeLong equality test. The odds ratios (OR) was calculated for CRP, albumin, and CAR according to the optimum cut-off values obtained from the ROC analysis. A p value lower than 0.05 was considered statistically significant in all analyses.

\section{Ethics}

Ethical approval for the study was obtained from the Ethics Committee of Ümraniye Training and Research Hospital (approval number: B.10. 1.TKH.4.34 .H.GP.0.01/113). We retrospectively reviewed the secondary data recorded in the computer-based patient data system of the hospital. The recorded data did not include any personally identifiable information, and it only consisted of clinical information. Therefore, informed patient consent was waived.

\section{Results \\ Patient characteristics}

Of the 103 patients included in the study, 57 (55.3\%) were male. The median of age of the 103 patients was 57 (25th-75th percentiles: 32-76) years. A total of five patients died within 30 days of ED presentation. The rate of 30-day mortality was $4.8 \%$ for the study cohort. The comparison of the demographic characteristics, clinical outcomes for the first 24 hours, comorbid diseases, symptoms, vital parameters at presentation, and mortality data between the survivor and non-survivor groups are shown in Table 1. The comparison of the initial laboratory findings between the two groups are presented in Table 2. Thirty-one patients (30.1\%) were discharged, 70 (67.96\%) and two $(1.94 \%)$ were admitted to the intensive care unit. Thirtyone of the patients who survived were discharged, while 67 were hospitalized. Three of the patients that died had been hospitalized, and two had been admitted to the intensive care unit. There was a statistically significant difference between the survivor and non-survivor groups in terms of the ED outcomes $(p=0.006)$.

\section{Laboratory values and outcomes}

Significant differences were observed between the survivor and non-survivor groups in relation to chronic renal failure [1 ( $1 \%$ ) versus $2(40 \%), \mathrm{p}=0.006]$, systolic blood pressure [128 (90-175) versus 165 (121-180), $\mathrm{p}=0.024]$, body temperature [36.6 (36-38.1) versus $37.5(37.2-39)$ per minute, $p=0.023$ ], and oxygen saturation [96 (85-99) versus $88(80-95) \%, p=0.007]$. There were also significant differences between the non-survivor and survivor groups in laboratory parameters: BUN [27.51 (10.68-61.9) versus $9.64(4.59-47.28) \mathrm{mmol} / \mathrm{L}, \mathrm{p}=0.008]$, creatinine $[0.43(0.29-3.36)$ versus $0.3(0.19-1.03) \mathrm{mmol} / \mathrm{L}, \mathrm{p}$ 

non-survivor groups

\begin{tabular}{|c|c|c|c|c|}
\hline Variables & Total & Survivor & Non-survivor & p values \\
\hline & $\begin{array}{l}n=103 \\
(\%, 25 \text { th }-75 \text { th percentiles })\end{array}$ & $\mathrm{n}=98(\%, 25$ th -75 th percentiles $)$ & $\mathrm{n}=5(\%, 25$ th -75 th percentiles $)$ & \\
\hline Age, years & $57(32-76)$ & $56(32-72)$ & $59(32-74)$ & 0.162 \\
\hline \multicolumn{4}{|l|}{ Gender } & \multirow{3}{*}{0.063} \\
\hline Male & $57(55.3 \%)$ & $52(53.1 \%)$ & $5(100 \%)$ & \\
\hline Female & $46(44.7 \%)$ & $56(56.9 \%)$ & 0 & \\
\hline \multicolumn{5}{|l|}{ Comorbidities } \\
\hline $\begin{array}{l}\text { Chronic obstructive pulmonary } \\
\text { diseases }\end{array}$ & $8(7.8 \%)$ & $7(7.1 \%)$ & $1(20 \%)$ & 0.338 \\
\hline Hypertension & $27(26.2 \%)$ & $25(25.5 \%)$ & $2(40 \%)$ & 0.604 \\
\hline Diabetes mellitus & $20(19.4 \%)$ & $19(19.4 \%)$ & $1(20 \%)$ & 0.999 \\
\hline Coronary artery disease & $7(6.8 \%)$ & $7(7.1 \%)$ & 0 & 0.999 \\
\hline Congestive heart failure & $4(3.9 \%)$ & $3(3.1 \%)$ & $1(20 \%)$ & 0.183 \\
\hline Chronic renal failure & $3(2.9 \%)$ & $1(1 \%)$ & $2(40 \%)$ & 0.006 \\
\hline \multicolumn{5}{|l|}{ Frequency of symptoms } \\
\hline Fever & $44(42.7 \%)$ & $41(41.8 \%)$ & $3(60 \%)$ & 0.649 \\
\hline Cough & $60(58.3 \%)$ & $58(59.2 \%)$ & $2(40 \%)$ & 0.647 \\
\hline Sputum & $1(1 \%)$ & $1(1 \%)$ & 0 & 0.999 \\
\hline Shortness of breath & $32(31.1 \%)$ & $31(31.6 \%)$ & $1(20 \%)$ & 0.999 \\
\hline Weakness & $23(22.3 \%)$ & $22(22.4 \%)$ & $1(20 \%)$ & 0.999 \\
\hline Muscle-joint pain & $12(11.7 \%)$ & $11(11.2 \%)$ & $1(20 \%)$ & 0.469 \\
\hline Loss of taste or smell & $4(3.9 \%)$ & $4(4.1 \%)$ & 0 & 0.999 \\
\hline Headache & $8(7.8 \%)$ & $8(8.2 \%)$ & 0 & 0.999 \\
\hline Sore throat & $12(11.7 \%)$ & $11(12.9 \%)$ & $1(20 \%)$ & 0.469 \\
\hline Nausea-vomiting & $9(8.7 \%)$ & $9(9.2 \%)$ & 0 & 0.999 \\
\hline Diarrhea & $3(2.9 \%)$ & $3(3.1 \%)$ & 0 & 0.999 \\
\hline \multicolumn{5}{|l|}{ Vital parameters } \\
\hline Pulse pressure & $86(59-180)$ & $86(59-145)$ & $113(83-180)$ & 0.061 \\
\hline Body temperature & $836.6(36-39)$ & $36.6(36-38.1)$ & $37.58(37.2-39)$ & 0.023 \\
\hline Respiratory rate & $20(16-27)$ & $20(16-27)$ & $18(17-19)$ & 0.192 \\
\hline Oxygen saturation & $96(80-99)$ & $96(85-99)$ & $88(80-95)$ & 0.007 \\
\hline
\end{tabular}

The data is presented as number and percent, median and 25 th to 75 th percentiles.

Table 2

Laboratory parameters of the enrolled patients and comparison of these parameters between the survivor and non-survivor groups

\begin{tabular}{|l|l|l|l|l|}
\hline Variables & Total & Survivor & Non-survivor & $\mathrm{p}$ values \\
\hline & Median $(25$ th- 75 th percentiles $)$ & Median $(25$ th- 75 th percentiles $)$ & Median $(25$ th- 75 th percentiles $)$ & \\
\hline Blood urea nitrogen, $\mathrm{mmol} / \mathrm{L}$ & $9.64(4.59-61,9)$ & $9.64(4.59-47.28)$ & $27.51(10.68-61.9)$ & $\mathbf{0 . 0 0 8}$ \\
\hline Creatinine, $\mathrm{mmol} / \mathrm{L}$ & $0.3(0.19-3.36)$ & $0.3(0.19-1.03)$ & $0.43(0.29-3.36)$ & $\mathbf{0 . 0 1 8}$ \\
\hline D-dimer, $\mu \mathrm{g} / \mathrm{mL}(\mathrm{DDU})$ & $625(230-4890)$ & $590(230-4,890)$ & $1748(1,530-1,966)$ & 0.062 \\
\hline Troponin $(\mathrm{cTnI}), \mathrm{ng} / \mathrm{mL}$ & $0.003(0.00-0.166)$ & $0.003(0.00-0.166)$ & $0.19(0.002-0.140)$ & $\mathbf{0 . 0 1 1}$ \\
\hline C-reactive $\mathrm{protein}, \mathrm{nmol} / \mathrm{L}$ & $28.57(9.52-209.52)$ & $28.57(9.52-209.52)$ & $85.71(19.04-200)$ & $\mathbf{0 . 0 2 0}$ \\
\hline Albumin, $\mathrm{mg} / \mathrm{dL}$ & $41.8(30.7-49.6)$ & $41.8(30.7-49.6)$ & $35.9(32.7-47.3)$ & 0.112 \\
\hline White blood cell count, $109 / \mathrm{L}$ & $7.6(1.9-15.1)$ & $8(5.1-10.3)$ & $5.3(1.9-15.1)$ & 0.846 \\
\hline Neutrophil count. $109 / \mathrm{L}$ & $5.16(0.1-20.52$ & $4.98(0.1-20.52)$ & $5.53(1.96-13.03)$ & 0.667 \\
\hline Lymphocyte count, $109 / \mathrm{L}$ & $1.58(0.42-4.52)$ & $1.59(0.42-4.4 .52)$ & $1.07(0.44-2.53)$ & 0.234 \\
\hline Platelet count, $109 / \mathrm{L}$ & $213(22-454)$ & $216(22-54)$ & $146(83-210)$ & $\mathbf{0 . 0 1 7}$ \\
\hline Mean platelet volume & $9.2(7.5-13.2)$ & $9.2(7.5-13.2)$ & $9.4(8.8-12.3)$ & 0.303 \\
\hline $\begin{array}{l}\text { C-reactive protein-to-albumin } \\
\text { ratio }\end{array}$ & $0.08(0.02-0.59)$ & $0.07(0.02-0.53)$ & $0.04-0.59)$ & $\mathbf{0 . 0 2 1}$ \\
\hline
\end{tabular}

$=0.018]$, cTnI [ $0.003(0.00-0.166)$ versus $0.19(0.002-0.140)$ $\mathrm{ng} / \mathrm{mL}, \mathrm{p}=0.011]$, CRP [85.71 (19.04-200) versus 28.57 (9.52209.52) nmol/L, $\mathrm{p}=0.020]$, platelet count $[216$ (22-54) versus $146(83-210) 109 / \mathrm{L}, \mathrm{p}=0.017]$, and CAR [ $0.07(0.02-0.53)$ versus $0.28(0.04-0.59) \mathrm{p}=0.021]$.

The ROC curve analysis was performed to determine the discriminative ability of laboratory parameters in 30-day mortality. Table 3 and Figure 1 present the cut-off values of
BUN, creatinine, cTnI, CRP, albumin, and CAR according to the best Youden's index, as well as their sensitivity, specificity, likelihood ratios (LR), AUC and 95\% confidence interval (CI) values. There was no statistically significant difference between the AUC values of the laboratory parameters (DeLong equality test, for CRP and CAR, CRP and BUN, CRP and creatinine, CRP and cTnI, CAR and BUN, CAR and creatinine, CAR and cTnI, BUN and creatinine, BUN and cTnI, and creatinine and cTnI; 


\begin{tabular}{|l|l|l|l|l|l|l|l|l|l|l|l|}
\hline & AUC & Cut-off & Sensitivity & Specificity & PPV & NPV & LR+ & LR- & Accuracy & $95 \%$ CI & p-value \\
\hline BUN & 0.849 & $>10$ & 100 & 55.1 & 10.2 & 100 & 2.23 & 0 & 55.1 & $76.5-91.2$ & $<0.001$ \\
\hline Creatinine & 0.815 & $>0.36$ & 80 & 76.5 & 14.8 & 98.7 & 3.41 & 0.26 & 56.53 & $72.7-88.5$ & 0.004 \\
\hline cTnI & 0.838 & $>0.01$ & 80 & 87.8 & 28.6 & 98.6 & 6.56 & 0.23 & 67.80 & $74.3-90.8$ & 0.002 \\
\hline CRP & 0.801 & $>66.67$ & 80 & 78.6 & 16 & 98,7 & 3.73 & 0,25 & 58.57 & $71.1-87.3$ & 0.003 \\
\hline Albumin & 0.711 & $\leq 35.9$ & 60 & 90.8 & 25 & 97.8 & 4.53 & 0.44 & 50.82 & $61.4-79.6$ & 0.203 \\
\hline CAR & 0.806 & $>0.18$ & 80 & 78.6 & 16 & 98.7 & 3.73 & 0.25 & 58.57 & $71.6-87.7$ & 0.005 \\
\hline
\end{tabular}

ED: emergency department; AUC: area under the curve; PPV: positive predictive value; NPV: negative predictive value; LR: likelihood ratio; CI: confidence interval; cTnI: high-sensitive cardiac troponin I, BUN: blood urea nitrogen; CRP: C-reactive protein; CAR: C-reactive protein-to-albumin ratio

Figure 1 - Receiver operating characteristic curves for C-reactive protein (CRP), C-reactive protein-to-albumin ratio (CAR), blood urea nitrogen (BUN), creatinine (Cre) and Troponin I (Trop) for the prediction of 30-day mortality in patients with COVID-19

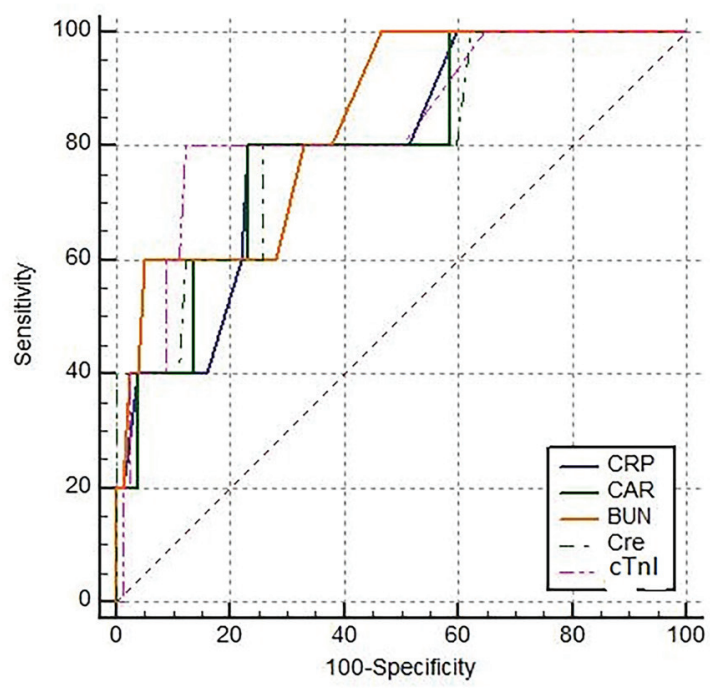

$\mathrm{p}$ values are $0.938(0.801$ versus 0.806$), 0.494(0.801$ versus $0.849), 0.987$ ( 0.801 versus 0.815$), 0.374(0.801$ versus 0.838$)$, 0.476 (0.806 versus 0.849$), 0.993$ (0.806 versus 0.815$), 0.326$ ( 0.806 versus 0.838$), 0.718(0.849$ versus 0.815$), 0.938(0.849$ versus 0.838$)$, and 0.833 (0.815 versus 0.838$)$, respectively). The OR of CRP $(>66.67 \mathrm{mg} / \mathrm{L})$, albumin $(<35.9 \mathrm{mg} / \mathrm{dL})$ and CAR $(>0.18)$ for 30-day mortality were determined as 14.667 (95\% CI: 1.555-138.299), 14.833 (95\% CI: 2.183-100.781), and 13.818 (95\% CI: 1.468-130.076), respectively.

\section{Discussion}

In this study, we examined the ability of CAR to predict short-term mortality in patient with COVID-19 presenting to ED. To the best of our knowledge, this is the first study that determines that CAR was not useful in predicting 30-day mortality in this patient group.

In our analysis, firstly, non-parametric comparison tests were used to determine the significant differences between the survivors and non-survivors in terms of the CRP, albumin, and CAR values. While CRP and CAR were significantly higher in the non-survivor group, no significant relationship was found between albumin and mortality. A further analysis was performed based on the ROC curve to control the ability of the two parameters to distinguish whether a patient survived or died. AUC values less than 0.5 were evaluated as indistinguishable from random, while those close to 1 were considered close to a perfect model $[9,10]$. In the literature, it has been reported that the AUC value should be greater than 0.8 for a model to predict mortality well $[9,10]$. In the discriminatory power analysis, we determined the AUC value of albumin as 0.711 , which was unacceptable. However, the AUC value of CRP in predicting 30day mortality was 0.801 , and that of albumin in predicting 30 day mortality was 0.801 , which indicated the predictive ability of this score for mortality. Thus, in this retrospective study, the ROC analysis confirmed that only CRP and CAR were predictors of 30-day mortality in patients with confirmed COVID-19. There was no significant difference between the AUC values of CPR and CAR. Furthermore, there was no significant difference between the ORs in the groups formed according to the cut-off values. Therefore, it is not clinically reasonable to calculate the CAR value when CRP can already predict short-term mortality according to the OR and ROC analysis. On the other hand, when LRs, which provide the clearest data on the way in which a scoring system can be used reliably [9-10], were examined, it was determined that CRP, albumin, and CAR could not be used clinically to predict short-term mortality in ED since their LR values were not in the range of below 0.2 or above 5 , which is accepted to indicate strong evidence [11-13].

CAR is a biomarker that has been studied as a prognostic marker in inflammatory and infectious diseases, and malignancy [14-15]. In the current literature, there are studies on the predictive ability of CAR in patients with COVID-19. In a study conducted by Karakoyun et al., CAR was reported as an independent predictor of severe disease in hospitalized patients [16]. In that study, the AUC values of CRP and CAR were reported as 0.697 and 0.718 , respectively, which do not represent a high predictive ability $[9,10,16]$. Furthermore, the OR for CAR in the prediction of severe disease was found to be 1.264, which is too low to be clinically significant [16]. Güney et al. investigated the predictive value of CAR for in-hospital mortality in patients with COVID-19 by dividing the cohort into three groups according to their CAR values (17). The authors found the OR as 8.2 between the group with a high CAR value and the reference group, which cannot be considered clinically significant $[13,17]$. El-Shabrawy et al. reported the AUC values as 0.939 and 0.955 for CRP and CAR, respectively and noted that these values were statistically significant in the prediction of 30-day mortality [18]. However, considering the very similar AUC values of the two parameters, we do not think that this clinical significance is sufficient to indicate that the calculation of CAR can replace that of CRP. In a study of Paliogiannis et al. with a small sample, no statistically significant difference was reported in terms of the CAR values between the survivor and non-survivor patient groups [19]. The results of our study also showed that CAR could not be used clinically to predict shortterm mortality in patients presenting to ED with COVID-19. A 
plausible explanation for this may be the decrease in albumin due to reasons such as prolonged hospitalization and malnutrition [17]. Therefore, the initial albumin value in the denominator may not have made a clinically significant contribution to the predictive ability of CRP.

\section{Limitations}

The main limitation of our study was its retrospective nature. Secondly, we were not able to include patients with COVID-19 who had not been tested for CRP and albumin. This was the most important factor limiting our study population. Lastly, our study had a single-center design, and therefore the results cannot be generalized to other healthcare institutions. We recommend multicenter studies in larger populations to confirm our results and increase their generalizability.

\section{Conclusion}

Based on the results of our study, CAR is a useful in predicting 30-day mortality in patients with COVID-19. Calculating CAR rather than CRP had no clinically significant contribution to the prediction of 30-day mortality in this patient group.

Disclosures: There is no conflict of interest for all authors.

\section{Acknowledgements: None.}

Funding: None.

\section{References}

1. Eroglu SE, Aksel G, Altunok I, et al. Can Google ${ }^{\circledR}$ trends predict emergency department admissions in pandemic periods? Medicine Science. 2021;10(1):111-117. https://doi.org/10.5455/medscience.2020.08.162

2. Sert E, Mutlu H, Kokulu K, Sarıtaş A. Anxiety Levels and Associated Factors Among Emergency Department Personnel Fighting COVID-19. J Contemp Med. 2020; 10(4): 556-561. https://doi.org/10.16899/jcm.972664

3. Zhou B, Kojima S, Kawamoto A, et al. COVID-19 pathogenesis, prognostic factors, and treatment strategy: Urgent recommendations. J Med Virol. 2021;93(5):2694-2704. https://doi.org/10.1002/jmv.26754.

4. Gallo Marin B, Aghagoli G, Lavine K, et al. Predictors of COVID-19 severity: A literature review. Rev Med Virol. 2021;31(1):1-10. https://doi.org/10.1002/rmv.2146.

5. Özdemir S, Eroğlu SE, Algin A, et al. Analysis of laboratory parameters in patients with COVID-19: Experiences from a pandemic hospital. Ann Clin Anal Med. 2021;12 (Supp 4):518-523. https://doi.org/10.4328/ACAM.20678

6. Aksel G, İslam MM, Algın A, et al. Early predictors of mortality for moderate to severely ill patients with Covid-19. Am J Emerg Med. 2021;45:290-296. https://doi.org/10.1016/j.ajem.2020.08.076.

7. Seyit M, Avci E, Nar R, et al. Neutrophil to lymphocyte ratio, lymphocyte to monocyte ratio and platelet to lymphocyte ratio to predict the severity of COVID-19. Am J Emerg Med. 2021;40:110-114. https://doi.org/10.1016/j.ajem.2020.11.058

8. Avramovski P, Avramovska M, Sotiroski K, Sikole A. Acute-phase proteins as promoters of abdominal aortic calcification in chronic dialysis patients. Saudi J Kidney Dis Transpl. 2019;30(2):376-386. https://doi.org/10.4103/1319-2442.256845.

9. Hanley JA, McNeil BJ. The meaning and use of the area under a receiver operating characteristic (ROC) curve. Radiology. 1982;143(1):29-36. https://doi.org/10.1148/radiology.143.1.7063747.

10. Hanley JA, McNeil BJ. A method of comparing the areas under receiver operating characteristic curves derived from the same cases. Radiology. 1983;148(3):839-43. https://doi.org/10.1148/radiology.148.3.6878708.

11. Deeks JJ. Systematic reviews of evaluations of diagnostic and screening tests. Egger M Davey Smith G Altman DG eds. Systematic reviews in health care: meta-analysis in context. Systematic reviews, 2nd ed 2001:248-282 BMJ Books London.

12. Price CP, Newall RG, Boyd JC. Use of protein: creatinine ratio measurements on random urine samples for prediction of significant proteinuria: a systematic review. Clin Chem. 2005;51(9):1577-86. https://doi.org/10.1373/clinchem.2005.049742.

13. Fischer JE, Bachmann LM, Jaeschke R. A readers' guide to the interpretation of diagnostic test properties: clinical example of sepsis. Intensive Care Med. 2003;29(7):1043-51. https://doi.org/10.1007/s00134-003-1761-8.

14. Özdemir S, Akça HŞ, Algın A, et al. Can C-reactive protein-to-albumin ratio be a predictor of short-term mortality in communityacquired pneumonia? Ann Clin Anal Med. 2021;12(9):1043-1048. https://doi.org/ 10.4328/ACAM.20576.

15. Acet H, Güzel T, Aslan B, et al. Predictive Value of C-Reactive Protein to Albumin Ratio in ST-Segment Elevation Myocardial Infarction Patients Treated with Primary Percutaneous Coronary Intervention. Angiology. 2021;72(3):244-251. https://doi. org/10.1177/0003319720963697.

16. Karakoyun I, Colak A, Turken M, et al. Diagnostic utility of C-reactive protein to albumin ratio as an early warning sign in hospitalized severe COVID-19 patients. Int Immunopharmacol. 2021;91:107285. https://doi.org/10.1016/j.intimp.2020.107285.

17. Güney BÇ, Taştan YÖ, Doğantekin B, et al. Predictive Value of CAR for In-Hospital Mortality in Patients with COVID-19 Pneumonia: A Retrospective Cohort Study. Arch Med Res. 2021;52(5):554-560. https://doi.org/10.1016/j.arcmed.2021.02.006.

18. El-Shabrawy M, Alsadik M, El-Shafei M, et al. Interleukin-6 and C-reactive protein/albumin ratio as predictors of COVID-19 severity and mortality. The Egyptian Journal of Bronchology. 2021;15(1). https://doi.org/10.1186/s43168-021-00054-1

19. Paliogiannis P, Zinellu A, Scano V, et al. Laboratory test alterations in patients with COVID-19 and non-COVID-19 interstitial pneumonia: a preliminary report. $J$ Infect Dev Ctries. 2020;14(7):685-690. https://doi.org/10.3855/jidc.12879. 